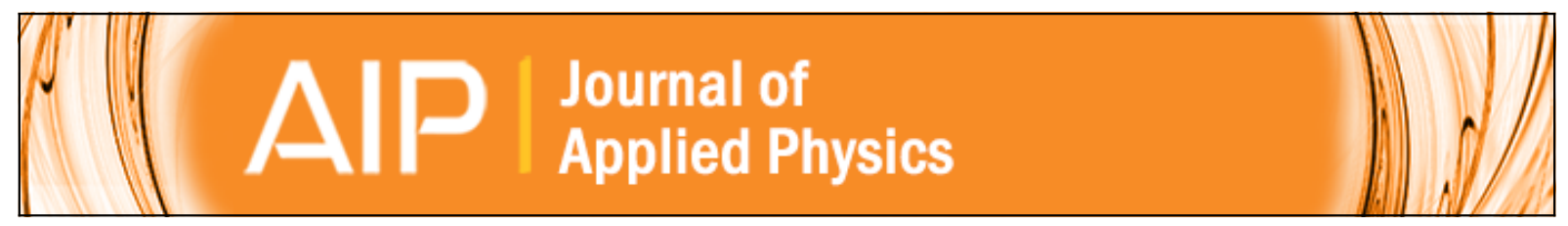

\title{
Brillouin scattering in planar waveguides. II. Experiments
}

A. Chiasera, M. Montagna, E. Moser, F. Rossi, C. Tosello, M. Ferrari, L. Zampedri, S. Caponi, R. R. Gonçalves,

S. Chaussedent, A. Monteil, D. Fioretto, G. Battaglin, F. Gonella, P. Mazzoldi, and G. C. Righini

Citation: Journal of Applied Physics 94, 4882 (2003); doi: 10.1063/1.1607520

View online: http://dx.doi.org/10.1063/1.1607520

View Table of Contents: http://scitation.aip.org/content/aip/journal/jap/94/8?ver=pdfcov

Published by the AIP Publishing

\section{AlP Re-register for Table of Content Alerts}

\section{Create a profile. \\ Sign up today!}




\title{
Brillouin scattering in planar waveguides. II. Experiments
}

\author{
A. Chiasera, M. Montagna, a) E. Moser, F. Rossi, and C. Tosello \\ Dipartimento di Fisica, Università di Trento and INFM, via Sommarive 14, I-38050 Trento, Italy \\ M. Ferrari and L. Zampedri \\ CNR-IFN, Istituto di Fotonica e Nanotecnologie, via Sommarive 14, I-38050 Trento, Italy \\ S. Caponi \\ Dipartimento di Fisica, Università dell'Aquila and INFM, via Betoio Coppito, I-67100 L'Aquila, Italy \\ R. R. Gonçalves \\ Istituto de Quimica-UNESP, P.O. Box 355, 14801-970-Araraquara, SP, Brazil
}

S. Chaussedent and A. Monteil

Laboratoire POMA, Unité Mixte de Recherche associée au CNRS 6136, Université d'Angers,

2 boulevard Lavoisier, 49045 Angers, France

D. Fioretto

Dipartimento di Fisica, Università di Perugia and INFM, via Pascoli, I-06123 Perugia, Italy

G. Battaglin and F. Gonella

Dipartimento di Chimica Fisica, Università di Venezia and INFM, Dorsoduro 2137, I-30123 Venezia, Italy

P. Mazzoldi

Dipartimento di Fisica, Università di Padova and INFM, via Marzolo 8, I-35131 Padova, Italy

G. C. Righini

CNR-IFAC Institute of Applied Physics "Nello Carrara," via Panciatichi 64, I-50127 Firenze, Italy

(Received 6 February 2003; accepted 17 July 2003)

\begin{abstract}
Silica-titania planar waveguides of different thicknesses and compositions have been produced by radio-frequency sputtering and dip coating on silica substrates. Waveguides were also produced by silver exchange on a soda-lime silicate glass substrate. Brillouin scattering of the samples has been studied by coupling the exciting laser beam with a prism to different transverse-electric (TE) modes of the waveguides, and collecting the scattered light from the front surface. In multimode waveguides, the spectra depend on the $m$ mode of excitation. For waveguides with a step index profile, two main peaks due to longitudinal phonons are present, apart from the case of the $\mathrm{TE}_{0}$ excitation, where a single peak is observed. The energy separation between the two peaks increases with the mode index. In graded-index waveguides, $m-1$ peaks of comparable intensities are observed. The spectra are reproduced very well by a model which considers the space distribution of the exciting field in the mode, a simple space dependence of the elasto-optic coefficients, through the value of the refraction index, and neglects the refraction of phonons. A single-fit parameter, i.e., the longitudinal sound velocity, is used to calculate as many spectra as is the number of modes in the waveguide. (C) 2003 American Institute of Physics. [DOI: 10.1063/1.1607520]
\end{abstract}

\section{INTRODUCTION}

Brillouin scattering, by excitation with visible light, is a powerful nondestructive technique for the measure of the sound velocity in bulk transparent glasses. ${ }^{1}$ Surface Brillouin scattering is extensively used in strongly absorbing materials or in thin films which cannot propagate the light. ${ }^{2-5}$ In this case, surface phonons, propagating along the plane and exponentially decaying along the direction perpendicular to the surface, are active in the inelastic scattering. In planar waveguides, Brillouin scattering by bulk phonons can be measured by coupling the exciting laser line to a transverseelectric (TE) or transverse-magnetic (TM) mode of the guide by a prism. ${ }^{6-11}$ The shape of the Brillouin spectra (BS) is

${ }^{\text {a)} E l e c t r o n i c ~ m a i l: ~ m o n t a g n a @ ~ s c i e n c e . u n i t n . i t ~}$ mode dependent: Two main peaks are produced by the longitudinal phonons and two by the transverse phonons, apart from the case of the $\mathrm{TE}_{0}$ excitation, which produces a single peak. ${ }^{11}$ This is because Brillouin scattering depends on the scattering angle and the light propagates in the waveguide at different angles for different modes, with a zig-zag path in a ray-tracing approach. A model for the calculation of the BS in a waveguide, valid for any refractive index profile, has been proposed in Ref. 12. This model makes provision for a rich phenomenology of line shapes in the case of gradedindex waveguides.

The main aim of this article is to test this model on some waveguides of different thicknesses and compositions, homogeneous or graded-index, produced by rf sputtering, sol gel, and ion mixing. The waveguides were characterized by scanning electron microscopy (SEM), Rutherford back- 
scattering (RBS), $m$-line technique, loss measurements, and Raman scattering. It is shown that a fit of the BS with the model of Ref. 12 allows the sound velocity of the waveguide to be measured with a good accuracy. This is an important characterization of a waveguide, as a preliminary step to optimizing the fabrication process in view of integrated optics applications. ${ }^{13-15}$ In fact, the measure of the sound velocity, together with that of the refractive index, can give information on possible porosity or nonstoichiometry of the waveguide. For instance, a residual small porosity, frequently present in sol-gel produced glasses, may strongly affect the sound velocity ${ }^{16,17}$ more than the refractive index. This is due to the fact that the mismatch of the refractive index is lower than that of the elastic constant, since the light propagates in the pores volume, whereas the acoustic waves cannot.

\section{EXPERIMENT}

\section{A. Sample preparation}

Silica-titania films were deposited by a rf sputtering technique, described in Ref. 18. Two samples, a silica slice $\left(25 \times 70 \mathrm{~mm}^{2}\right)$ and a silicon slice, were coupled on the sample holder: The films deposited on silicon were used for performing SEM and RBS measurements. The as-prepared films on the silica substrate did not propagate the light because of nonstoichiometric structure of $\mathrm{SiO}_{x}$ with $x<2$, and thermal annealing in air for $6 \mathrm{~h}$ at $600^{\circ} \mathrm{C}$ was necessary to achieve light propagation. The axis of circular symmetry of the sputtering apparatus was at the middle point of the long side border between the silica and silicon substrates. Due to the geometry of the plasma generated by circular magnetron, the thickness of the deposited films decreases from the axis toward the borders of the sample holder. This effect is evidenced by the appearance of circular interference fringes on the films. For instance, the thickness of sample RFSW1, measured by a $m$-line technique, decreases from about 2.8 $\mu \mathrm{m}$ to $2.65 \mu \mathrm{m}$ going from near one border side of the slice to the other one, along a $15 \mathrm{~mm}$ length.

The sample RFSW1, used for photonics applications, was activated with $1 \mathrm{~mol} \% \mathrm{Er}^{18,19}$ The sample RFSW2 was produced with a higher erbium content. Due to an inhomogeneous concentration of erbium caused by a progressive increase of the sputtering efficiency of the erbium wire, which was initially oxidized, a graded-index waveguide was produced. This effect was negligible for the RFSW1 waveguide, having a lower erbium content.

Silica-titania sol-gel samples were deposited by a dipcoating technique, described in Ref. 20. The graded-index $\mathrm{SiO}_{2}-\mathrm{TiO}_{2}$ planar waveguide (SGW1) was obtained by subsequently depositing 35 layers of $8 \% \mathrm{TiO}_{2}-92 \% \mathrm{SiO}_{2}$ composition, 25 layers of $16 \% \mathrm{TiO}_{2}-84 \% \mathrm{SiO}_{2}$, and finally 23 layers of $24 \% \mathrm{TiO}_{2}-76 \% \mathrm{SiO}_{2}$ composition, on a silica substrate $\left(70 \times 25 \times 1 \mathrm{~mm}^{3}\right)$. In parallel, three single-layered planar waveguides (R1, R2, and R3) were also produced, for reference, with the same composition and experimental procedure. After each dip, the films were annealed in air at $900{ }^{\circ} \mathrm{C}$ for $30 \mathrm{~s}$. After every ten dipping cycles, the films were heat treated at $900{ }^{\circ} \mathrm{C}$ for 2 min. Finally, the waveguides were submitted to a further heat treatment at $1000^{\circ} \mathrm{C}$ with a heating rate from 600 to $1000^{\circ} \mathrm{C}$ of $20^{\circ} \mathrm{C} /$ $\min$.

Waveguides were also produced by silver exchange on a soda-lime silicate glass substrate. Samples were obtained by melting, at $1400^{\circ} \mathrm{C}$, the base oxides with different weight concentrations; the glass used for the fabrication of the IEW1 waveguide had the composition $68.8 \mathrm{SiO}_{2}-2.0 \mathrm{Al}_{2} \mathrm{O}_{3}-1.0$ $\mathrm{P}_{2} \mathrm{O}_{5}-14.8 \quad \mathrm{Na}_{2} \mathrm{O}-1.0 \quad \mathrm{~K}_{2} \mathrm{O}-9.4 \mathrm{CaO}-3.0 \quad \mathrm{Er}_{2} \mathrm{O}_{3}$. The glasses were cut in $35 \times 25 \times 1 \mathrm{~mm}^{3}$ plates. The planar waveguides were obtained by immersing the plates for different times, of the order of $1 \mathrm{~h}$, in a molten salt containing $0.5 \mathrm{AgNO}_{3}$ and $99.5 \mathrm{NaNO}_{3} \mathrm{~mol} \%$ at $325^{\circ} \mathrm{C}$, in order to produce waveguides of different thicknesses.

\section{B. Sample characterization}

SEM was used to analyze the morphology of both the substrates and the waveguiding films. The surface of the films was analyzed by a JEOL-JSM 6300 apparatus at $15 \mathrm{kV}$ by covering the films with a $20 \mathrm{~nm}$ gold layer. Energy dispersive spectrometry (EDS) was performed in order to achieve a compositional analysis, by using a Noran Instruments mod. Voyager apparatus. In order to obtain more accurate values for the concentration of the different atomic species, some samples were analyzed by RBS. RBS analyses were performed at INFN National Labs of Legnaro (Padova) using a $2.2 \mathrm{MeV}^{4} \mathrm{He}^{+}$beam at normal incidence; scattered particles were detected at an angle of $160^{\circ}$.

The refractive index and the thickness of the waveguides were measured for both TE and TM polarization by an $m$-line apparatus (Metricon mod. 2010) based on the prism coupling technique. We used a gadolinium gallium garnet prism with a refractive index 1.9644 at $632.8 \mathrm{~nm}$. The apparatus was equipped with a $\mathrm{Si}$ detector to collect the visible light. Two He-Ne lasers, operating at 632.8 and $543.5 \mathrm{~nm}$, were employed. The resolution in the determination of the angles synchronous to the propagation modes was $0.0075^{\circ}$.

In order to measure propagation losses, the light intensity scattered out of the waveguide plane, which is proportional to the guided intensity, was recorded by a video camera. The losses were evaluated by fitting the intensity to an exponential decay function, assuming a homogeneous distribution of the scattering centers in the film. ${ }^{21}$ The measurements were performed by exciting the transverse electric $\mathrm{TE}_{0}$ mode of the waveguide with a $\mathrm{He}-\mathrm{Ne}$ laser operating at $632.8 \mathrm{~nm}$.

\section{Brillouin scattering}

BS spectra were measured by using an $\mathrm{Ar}^{+}$laser, operating at a power of about $250 \mathrm{~mW}$ on a single mode of the $514.5 \mathrm{~nm}$ line, coupled to a $\mathrm{TE}_{m}$ mode of the waveguide by a prism. The scattered light was collected from the front surface in a direction nearly perpendicular to the planar waveguide. A $\mathrm{He}-\mathrm{Ne}$ laser, coaxial with the detection axis allowed the actual orientation of the waveguide to be measured, because the orientation changed with the mode index $m$. A SOPRA double monochromator with gratings operating at the 11th order was employed. This slits were placed in 
order to have a typical frequency resolution of $0.03-0.06$ $\mathrm{cm}^{-1}$. Some BS were measured by means of a Sandercocktype $(3+3)$-pass tandem Fabry-Perot interferometer characterized by a finesse approximately 100 and a contrast ratio greater than $5 \times 10^{10}{ }^{3}$ A single-mode diode pumped solidstate laser operating at $532 \mathrm{~nm}$ was used with a power of about $250 \mathrm{~mW}$. The instrumental resolution function was estimated by the half width at half maximum of the elastic peak and it proved to be around $0.013 \mathrm{~cm}^{-1}$.

The low resolution of the Sopra apparatus was sufficient, except for the case of the spectra of the RFS2 waveguide. In this case, the use of the higher-resolution Fabry-Perot apparatus was really crucial to detect the structured BS.

\section{RESULTS AND DISCUSSION}

\section{A. Homogeneous waveguides}

The refractive index profile of the RFSW1 planar waveguide, reconstructed from the effective indices at $543.5 \mathrm{~nm}$ by an inverse Wentzel-Kramer-Brillouin (WKB) method, ${ }^{22}$ is similar for TE and TM polarizations, indicating that the birefringence in this waveguide is negligible. The refractive index along the thickness is sufficiently uniform, indicating a good homogeneity of the film. In fact, the six effective indices, for the three TE and three TM modes, are reproduced within a $\Delta n<0.001$ by a single-step profile with $n_{g}=1.493$ and thickness $t=2.8 \mu \mathrm{m}$. These data were obtained by coupling the waveguide in a region near the maximum thickness (the center of the circular fringes). The further the coupling from this region, the lower the effective indices. All data are consistent with a constant refractive index of the film $n_{g}$ $=1.493$ and with a thickness which decreases by about $5 \%$ (from $t=2.80 \mu \mathrm{m}$ to $t=2.65 \mu \mathrm{m}$ ) at $1.5 \mathrm{~cm}$ from the center of the rings. At $514.5 \mathrm{~nm}$, a fourth mode appears in TE polarization (not in TM), but this occurs only in the region where the thickness is greater than about $2.7 \mu \mathrm{m}$. To calculate the electric-field patterns of the modes, we need the refractive index at $514.5 \mathrm{~nm}$; the wavelength used in Brillouin spectroscopy. The value $n_{g}(514.5 \mathrm{~nm})=1.4975$ is obtained by extrapolation from the data $n_{g}(543.5 \mathrm{~nm})=1.493$ and $n_{g}(632.8 \mathrm{~nm})=1.489$. Since the dispersion curves of mixed $\mathrm{SiO}_{2}-\mathrm{TiO}_{2}$ systems are not available, the extrapolation procedure could be affected by a serious error.

EDS measurements on the film deposited on silicon substrate, reference of the RFSW1 waveguide, give a 92\% $\mathrm{SiO}_{2}-7 \% \quad \mathrm{TiO}_{2}-1 \% \quad \mathrm{ErO}_{2 / 3}$ composition, with $10 \%$ estimated error. The measured refractive index of the waveguide, at $632.8 \mathrm{~nm}$, is reproduced very well by the LorentzLorenz equation for this composition, using $n=1.457$ and $n=2.385$ for the refractive index of $\mathrm{SiO}_{2}$ and $\mathrm{TiO}_{2}$, respectively. This indicates that the film has no important residual porosity.

An attenuation coefficient of $0.2 \mathrm{~dB} / \mathrm{cm}$ is measured at $632.8 \mathrm{~nm}$ for the $\mathrm{TE}_{0}$ mode of the RFSW1 waveguide. The main source of losses in this kind of waveguide is the surface roughness. ${ }^{23}$ By SEM measurements, no surface structure is evidenced. The losses are low also at $514.5 \mathrm{~nm}$, so that a nearly constant excitation is present along the path of about 5

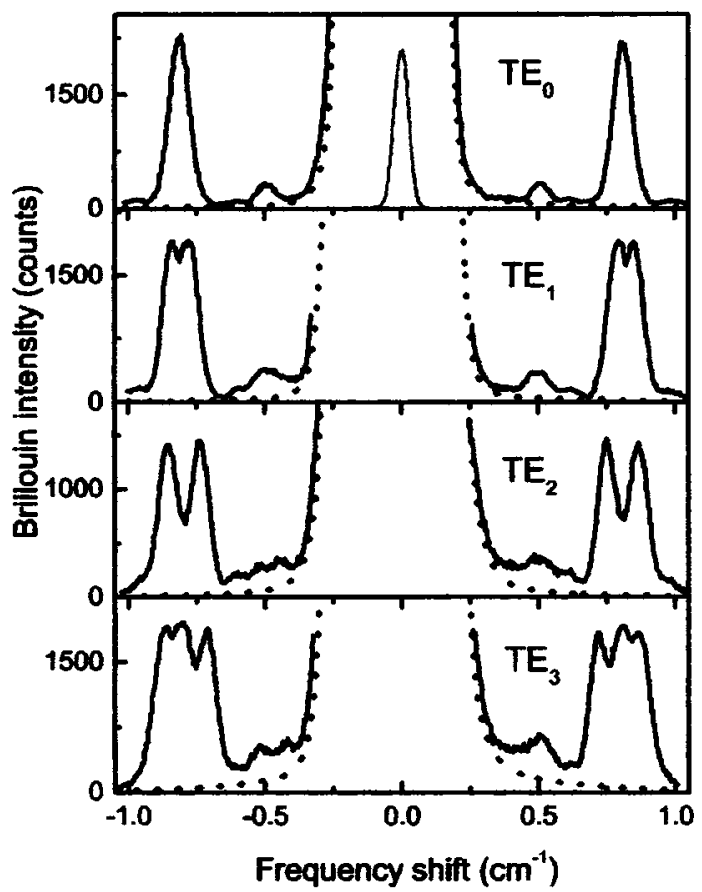

FIG. 1. BS of the RFSW1 waveguide, excited at $514.5 \mathrm{~nm}$ in the four $\mathrm{TE}_{m}$ modes. The scattering from transverse and longitudinal phonons appears in the spectral regions at about $0.5 \mathrm{~cm}-1$ and $0.8 \mathrm{~cm}-1$, respectively. The elastic peak of the $\mathrm{TE}_{0}$ spectrum, reduced in intensity by a factor of about $10^{4}$, shows the frequency resolution $(0.055 \mathrm{~cm}-1)$ of the measure. The dotted lines are an estimation of the elastic tail, to be subtracted from the spectra.

$\mathrm{mm}$ of the guided light, seen by the collection optics of the Raman or Brillouin spectrometers.

Figure 1 shows the BS of the RFSW1 waveguide, excited in the four $\mathrm{TE}_{m}$ modes. Two well defined peaks at about $0.5 \mathrm{~cm}^{-1}$ and $0.8 \mathrm{~cm}^{-1}$ appear in the $\mathrm{TE}_{0}$ spectra, due to the scattering from transverse and longitudinal phonons, respectively. They are relatively well resolved from the tails of the elastic scattering both in the Stokes and in the antiStokes parts of the spectrum. The elastic peak, reduced in intensity by a factor of about $10^{4}$, is also reported in order to show the frequency resolution of the measure $(\Delta \nu$ $=0.055 \mathrm{~cm}^{-1}$ ). The width of Brillouin peaks is larger than the frequency resolution of the apparatus.

The two weak peaks centered at about $0.6 \mathrm{~cm}^{-1}$ and at $1.0 \mathrm{~cm}^{-1}$ are spurious and their intensity depends on the quality of the prism waveguide coupling. They are due to substrate modes produced by light scattered at the prism waveguide coupling region: They dominate the spectra obtained by detuning the waveguide from the guiding angle on both sides, when the waveguide is not excited and a quite weak intensity enters into the substrate. These two spurious peaks are present in all spectra, but their intensity is usually weak and they are often confused in the background.

For $\mathrm{TE}_{1}$ excitation, the longitudinal and the transverse peaks split into two components, even if the splitting of the transverse peak is not really well resolved. The splitting increases as the modal number increases. The origin of this splitting, discussed in detail in Ref. 12, is easily explained by a simple ray-tracing approach, which considers that scatter- 


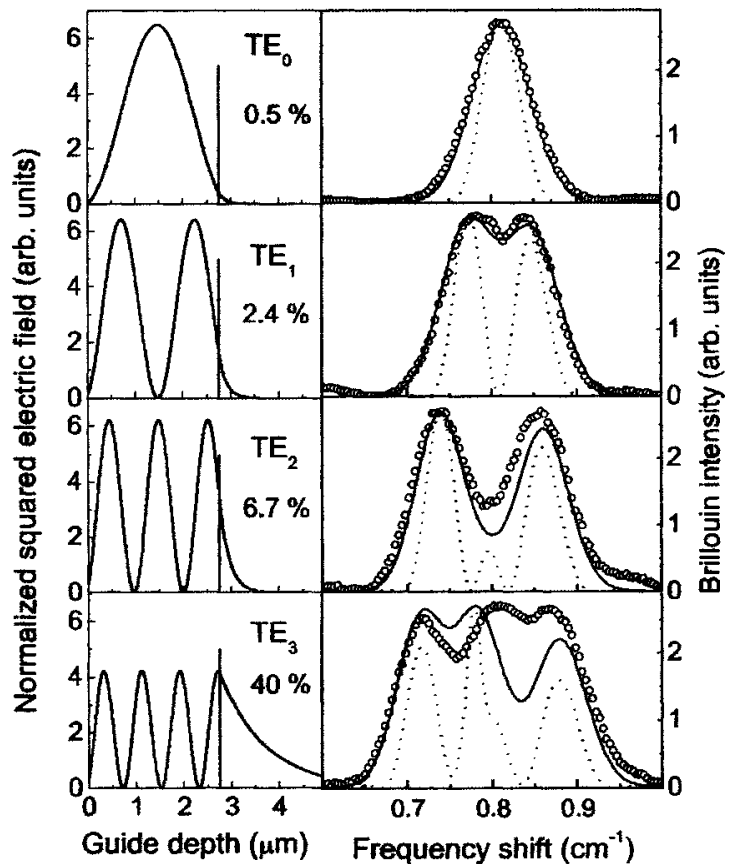

FIG. 2. Left-hand side column: Calculated squared electric-field patterns of the $\lambda=514.5 \mathrm{~nm} \mathrm{TE}{ }_{m}$ excitations as a function of the depth in the RFSW1 waveguide. The vertical bar indicates the position of the film-substrate interface. The percentage of the laser intensity traveling in the evanescent tail of the substrate is indicated. Right-hand side column: BS in the frequency region of the longitudinal phonons after subtraction of the elastic tail and of the estimated transverse contribution and averaging the Stokes and antiStokes spectra (open circles); spectra calculated by the model (of Ref. 12) (dotted line) with $v_{L}=5.88 \mathrm{~km} / \mathrm{s}$ for $\mathrm{TE}_{0}$ excitation, $v_{L}=5.90 \mathrm{~km} / \mathrm{s}\left(\mathrm{TE}_{1}\right)$, $v_{L}=5.86 \mathrm{~km} / \mathrm{s}\left(\mathrm{TE}_{2}\right)$, and $v_{L}=5.90 \mathrm{~km} / \mathrm{s}\left(\mathrm{TE}_{3}\right)$; convolution of the calculated spectra with the instrumental response (solid line).

ing occurs at two different exchanged $\mathbf{q}$ values, corresponding to the two directions of propagation of the laser beam in its zig-zag path within the waveguide. ${ }^{11}$ The interference of the two scattered fields causes the appearance of a single peak in the case of $\mathrm{TE}_{0}$ excitation.

For $m=2,3$, this splitting is large enough to cause a partial superimposition of the longitudinal and transverse parts of the spectrum; furthermore, the low-energy part of the transverse contribution is no longer well resolved from the elastic tail. The dotted lines in Fig. 1 are an estimation of this tail, to be subtracted from the spectra. After this subtraction, the anti-Stokes and Stokes spectra are compared to check intensities and frequency position of the peaks. The intensities should be the same, since the Bose factor $n(\omega, T)$, at room temperature and for $\omega \leqslant 1 \mathrm{~cm}^{-1}$, is much larger than 1.0. The peak positions, after the corrections for the nonlinear scan of the apparatus, are reproduced, from scan to scan, within $0.01 \mathrm{~cm}^{-1}$. This limits the accuracy of the measure of the longitudinal sound velocity to about $1 \%$ ( $2 \%$ for the transverse one). After this comparison, the average of Stokes and anti-Stokes spectra is considered. Figure 2 shows the result of this analysis in the longitudinal phonon spectral region, after a further subtraction of the transverse phonon contribution for the $\mathrm{TE}_{2}$ and $\mathrm{TE}_{3}$ spectra. The experimental spectra are compared with the results of the calculation using the model of Ref. 12. Here, and in the following, we will use for the polarizability tensor the form $\Pi_{y y}(X) \propto\left(n^{2}(X)-1\right)$, where $n(X)$ is the refractive index at a depth $X$ from the surface of the film. For the waveguide under study, $n(X)$ $=1.4975$ for $X<2.75 \mu \mathrm{m}$ and $n(X)=1.4617$, the value of the silica substrate, for $X>2.75 \mu \mathrm{m}$. The convolution of the calculated spectra with the spectral response of the apparatus is also shown, in order to allow a direct comparison with the experimental spectra. The calculated spectra were stretched in both directions: On the intensity scale, as usual, but also on the frequency scale. This corresponds to adjusting the sound velocities to fit the frequencies of the peaks. In this way, the values of the longitudinal and transverse sound velocities are obtained from each spectrum. The model reproduces very well the $\mathrm{TE}_{0}$ and $\mathrm{TE}_{1}$ spectra while the agreement, in the case of the $\mathrm{TE}_{2}$ spectrum, is not as close. The $\mathrm{TE}_{3}$ spectrum shows three peaks. The position of the central peak is not reproduced by the model. This point will be discussed later.

Within the uncertainty due to the limited reproducibility of the peak frequencies, the value of the sound velocities are the same for all $m, v_{L}=(5.88 \pm 0.06) \mathrm{km} / \mathrm{s}$, and $v_{T}=(3.63$ $\pm 0.05) \mathrm{km} / \mathrm{s}$.

A detailed analysis of the four experimental spectra of Fig. 2 shows that the center of mass of both the longitudinal and transverse contributions shifts slightly toward lower frequencies as the mode index $m$ increases. In fact, the waveguide is positioned nearly perpendicular to the axis of light detection and turned at different angles in order to couple the exciting laser beam to the four modes. Therefore, the scattered light is not really collected exactly at $90^{\circ}$ degrees, but at angles between $91^{\circ}$ for the $\mathrm{TE}_{0}$ excitation and $88.5^{\circ}$ for $\mathrm{TE}_{3}$ excitation. Of course, the calculated spectra take into account the actual orientation of the waveguide.

As shown on the left-hand side of Fig. 2, the three $\mathrm{TE}_{m}$ modes with $m \leqslant 2$ are very well confined in the waveguide, but this is not the case for the $\mathrm{TE}_{3}$ mode which extends deeply into the substrate with an important evanescent tail. Therefore, the $m \leqslant 2$ BS gain intensity only on the guiding film and indeed give the right information on their elastic properties. On the contrary, the $\mathrm{TE}_{3}$ spectrum has an important contribution deriving from the silica substrate. In this case, the measured sound velocities are effective values, with different weights from the substrate and the film. This is better clarified in Fig. 3 by the comparison of the $\mathrm{TE}_{3}$ spectra taken by coupling the prism to different regions of the waveguides, corresponding to different values of thickness $t$. In each measurement, the excited volume had a nearly equivalent thickness, because the waveguide was oriented on the coupling prism in order to have the laser beam tangent to the interference rings produced by the thickness gradient. A small change in the thickness has dramatic effects on the field profile of the $\mathrm{TE}_{3}$ mode and, therefore, on the BS, the more important the evanescent field in the substrate, the more intense the central peak with respect to the two other external peaks. In fact, the external peaks are produced by the two beams which propagate with different wave vectors, and with a definite phase relation, causing interference, in the zig-zag path within the waveguide. ${ }^{12}$ The central peak, on the contrary, is due to the scattering of the substrate, excited by the evanescent field. Since the model assumes a single sound 


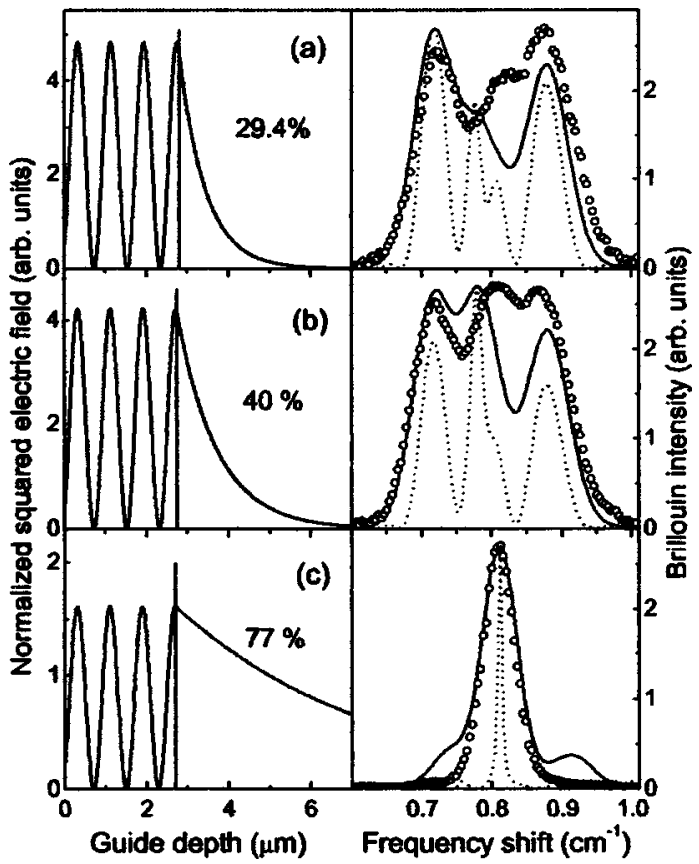

FIG. 3. Left-hand side column: Squared electric-field patterns of the $\lambda$ $=514.5 \mathrm{~nm} \mathrm{TE}{ }_{3}$ excitations as a function of the depth in the RFSW1 waveguide, calculated for three different values of the thickness. The vertical bar indicates the position of the film-substrate interface at $2.8 \mu \mathrm{m}$ (a), $2.75 \mu \mathrm{m}$ (b), and $2.7 \mu \mathrm{m}$ (c). The percentage of the laser intensity traveling in the evanescent tail of the substrate is indicated. Right-hand side column: Estimated BS of the longitudinal phonons (open circles); spectra calculated with $v_{L}=5.88 \mathrm{~km} / \mathrm{s}[(\mathrm{a})$ and (b) $]$ and $v_{L}=6.1 \mathrm{~km} / \mathrm{s}$ (c) (dotted line) and convolution of the calculated spectra with the instrumental response (solid line).

velocity for the waveguide-substrate system, it cannot reproduce the position of the two contributions at the same time. The frequencies of the two external peaks are reproduced by a longitudinal sound velocity (of the waveguide) $v_{L}$ $=5.88 \mathrm{~km} / \mathrm{s}$ [Figs. 3(a) and 3(b)], while the frequency of the central peak is reproduced by a longitudinal sound velocity $v_{L}=6.10 \mathrm{~km} / \mathrm{s}$ [Figs. 3(c)]. This value is close to that of the sound velocity of the substrate, $v_{L}^{S}=(6.05 \pm 0.06) \mathrm{km} / \mathrm{s}$. Note that the scattered intensity at any frequency is obtained by squaring the sum of the fields scattered at any point in the system, i.e., waveguide and substrate. Therefore, the aforementioned attributions should be considered as purely indicative, since the interference of the two contributions is important. However, the spectrum of Fig. 3(c) is very similar to that of the bulk substrate, measured in a standard $90^{\circ}$ geometry. As a matter of fact, in this case, most of the intensity $(77 \%)$ of the laser beam travels in the substrate. The width of the Brillouin line in the substrate spectrum (not shown) is governed only by the frequency response of the apparatus since the excitation beam has a large waist (about $50 \mu \mathrm{m}$ ) in this experiment; the width of the peak of Fig. 3(c) is greater, because the excitation exponentially decreases in the substrate (half of the intensity is reached at a depth $d$ $=3.3 \mu \mathrm{m}$ from the waveguide-substrate interface), so that phonons producing scattering do not have a well defined wave vector component along the direction perpendicular to the waveguide surface $\left(\Delta k_{X} \simeq 1 / d\right)$.

From the analysis of the RFSW1 waveguide, we conclude: (i) the measured sound velocities, $v_{L}=(5.88$

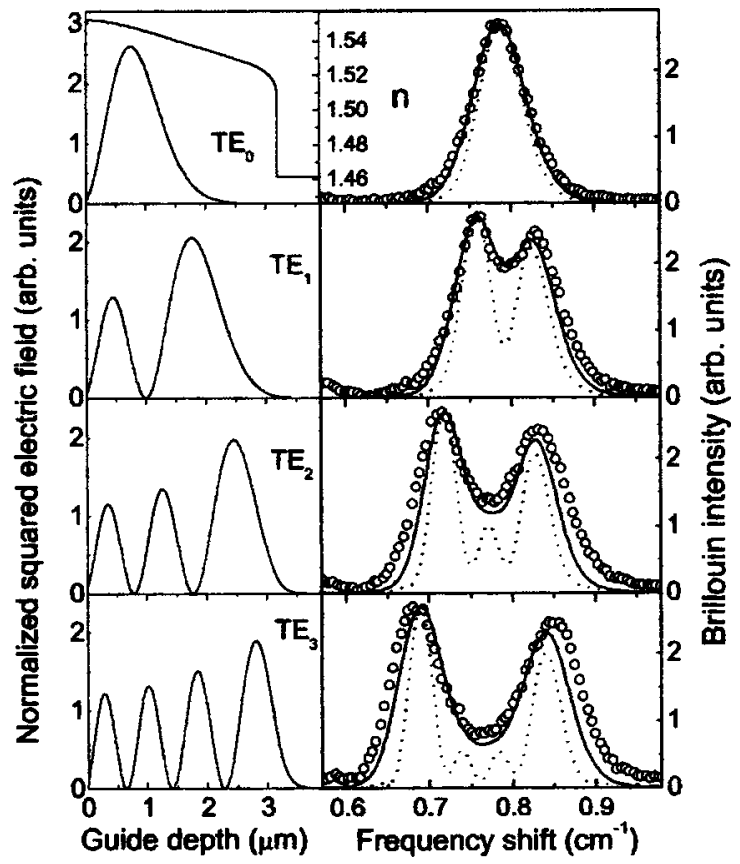

FIG. 4. Upper left-hand side frame: Refractive index profile at $514.5 \mathrm{~nm}$ of the RFSW2 waveguide, reconstructed from modal measurements at 543.5 $\mathrm{nm}$ and at $632.8 \mathrm{~nm}$ for the TE polarization. Left-hand side column: calculated squared electric-field patterns of the five $\mathrm{TE}_{m}$ modes at $514.5 \mathrm{~nm}$. Right-hand side column: estimated Brillouin spectra of the longitudinal phonons (open circles); spectra calculated by the model (of Ref. 12) with $v_{L}=5.6 \mathrm{~km} / \mathrm{s}$ (dotted line) and convolution of the calculated spectra with the instrumental response (solid line).

$\pm 0.06) \mathrm{km} / \mathrm{s}$ and $v_{T}=(3.63 \pm 0.05) \mathrm{km} / \mathrm{s}$, are slightly greater than those measured in Ref. 11. This could be attributed to the higher-temperature annealing $\left(T_{a}=600^{\circ} \mathrm{C}\right.$ in the present case, $T_{a}=400^{\circ} \mathrm{C}$ in the case of Ref. 11), which improves the stoichiometry and reduces the losses by producing a more compact structure; (ii) the model of Ref. 12 reproduces the observed BS not only for well confined mode, but also for modes with an important evanescent field in the substrate, even if the two fit with different values of the sound velocities of the waveguide and substrate and are needed for reproducing the two contributions.

\section{B. Graded-index waveguides}

Figure 4 shows the BS of a graded-index planar waveguide (RFSW2) in the region of the longitudinal phonons. The upper left-hand side of Fig. 4 shows the refractive index profile obtained at $514.5 \mathrm{~nm}$ by extrapolation of those calculated by a WKB inverse method at $543.5 \mathrm{~nm}$ and $632.8 \mathrm{~nm}$, based on the $m$-line data. RBS data indicate that the silica/ titania ratio is constant across the waveguide $(10 \%$ $\mathrm{TiO}_{2}-90 \% \quad \mathrm{SiO}_{2}$ ). On the contrary, a gradient of erbium concentration is measured. The ratio $\mathrm{Er} /(\mathrm{Si}+\mathrm{Ti})$ increases from about $1.4 \mathrm{~mol} \%$ near the substrate, to about $2.9 \mathrm{~mol} \%$ at the surface of the film. The observed gradient of the refractive index can be ascribed to the erbium concentration gradient. ${ }^{24}$ The squared electric-field patterns of four $\mathrm{TE}_{m}$ modes (the waveguide has six TE modes at $514.5 \mathrm{~nm}$ ) are also shown on the left-hand side of Fig. 4. The right-hand column of Fig. 4 shows the corresponding BS, measured by 


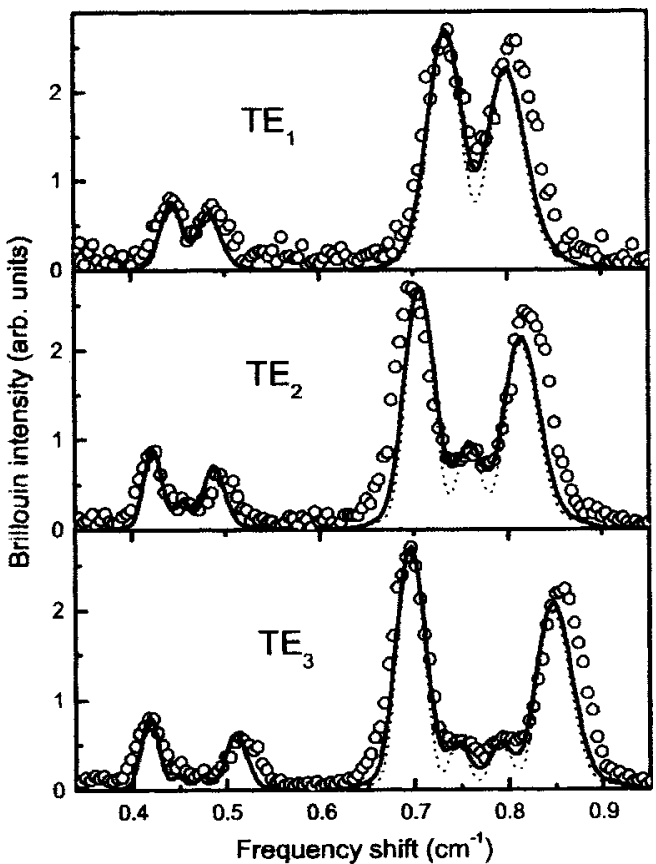

FIG. 5. BS (open circles) of the RFSW2 waveguide by excitation at $532 \mathrm{~nm}$ after averaging the Stokes and anti-Stokes spectra; spectra calculated by the model (of Ref. 12) using $v_{L}=5.6 \mathrm{~km} / \mathrm{s}$ and $v_{T}=3.37 \mathrm{~km} / \mathrm{s}$ for the $m=1,2$ spectra and $v_{L}=5.74 \mathrm{~km} / \mathrm{s}$ and $v_{T}=3.45 \mathrm{~km} / \mathrm{s}$ for the $m=3$ spectrum (dotted line) and convolution of the calculated spectra with the instrumental response (solid line).

the SOPRA apparatus, together with the results of the model of Ref. 12 (dotted line). For $\mathrm{TE}_{m}$ excitations with $m \geqslant 2$, additional peaks between the two main longitudinal peaks appear in the calculated spectra. However, the minor structures are lost after convolution of the calculated spectra with the response function of the SOPRA spectrometer (solid line in Fig. 4). Higher-resolution measurements are indeed to possibly evidence the provisions made by the model. Figure 5 shows the BS for the modes $m=1,2,3$ taken with higher resolution $\left(0.013 \mathrm{~cm}^{-1}\right)$ by using a tandem Fabry-Perot. This apparatus employs a single-mode diode pumped solidstate laser operating at $532 \mathrm{~nm}$ and, therefore, the data of Figs. 4 and 5 are not directly comparable. Of course, also the field profiles of Fig. 5 (not reported; at $532 \mathrm{~nm}$ there are only five TE modes) and the calculated BS are slightly different from those of Fig. 4. The experimental resolution is now sufficient to detect the central peaks in the $\mathrm{TE}_{2}$ and $\mathrm{TE}_{3}$ spectra, as shown by the convolution of the calculated spectra with the response function of the Fabry-Perot (solid line in Fig. 5). As a matter of fact, the central structures, even if not really well resolved, seem to appear, but the observed structures are broader than the calculated ones. The observed splittings are larger than the calculated ones and the relative intensities of the two main peaks of the longitudinal phonons are not well reproduced by the model. In this case, the comparison of the experimental spectra with those calculated by the model of Ref. 12 is quite critical since the model does not reproduce the observed line shape very well. The lack of a full agreement is the main source of error in the estimated sound velocities. For $\mathrm{TE}_{3}$ excitation, both the transverse $\left(v_{T}=3.45 \pm 0.04 \mathrm{~km} / \mathrm{s}\right)$ and longitudinal $\left(v_{L}=5.74\right.$ $\pm 0.04 \mathrm{~km} / \mathrm{s}$ ) sound velocities appear to be slightly higher than those used for fitting the spectra of other excitations $\left(v_{T}=3.37 \pm 0.04 \mathrm{~km} / \mathrm{s}, v_{L}=5.60 \pm 0.04 \mathrm{~km} / \mathrm{s}\right)$. This could be related to a gradient in the elastic constant profile caused by the gradient in the erbium content.

\section{Sol-gel waveguides}

A more stringent test of the Brillouin scattering model for the graded-index waveguides has been performed by producing a three-layered $\mathrm{SiO}_{2}-\mathrm{TiO}_{2}$ waveguide by the sol-gel dip-coating technique. The waveguide (SGW1) supports five TE and TM modes at $543.5 \mathrm{~nm}$ and four at $632.8 \mathrm{~nm}$. Three single-layered waveguides (R1, R2, and R3), with the same composition and growth procedure, were also produced for reference. The composition of the three layers was $8 \% \mathrm{TiO}_{2}-92 \% \mathrm{SiO}_{2}, \quad 16 \% \mathrm{TiO}_{2}-84 \% \mathrm{SiO}_{2}, \quad$ and $24 \% \mathrm{TiO}_{2}-76 \% \mathrm{SiO}_{2}$, respectively, starting from the substrate. The thickness and refractive index of the three reference waveguides, measured by $m$-line technique, are reported in Table I. The graded-index and the three singlelayered waveguides were characterized by Raman scattering. The comparison of the Raman spectra taken by excitation in different modes of the graded-index waveguide, allows one to selectively study the three layers. ${ }^{25}$ From this study, the structure of the second and the third layer appears to be the same as that of the reference samples, but this is not the case for the first layer with the lowest titania concentration. The final thermal annealing at $1000{ }^{\circ} \mathrm{C}$ produced complete densification in the reference waveguide, but a residual porosity was present in the first layer of the graded-index waveguide. This was probably due to a densification of the external layers occurring at a lower temperature than that of the internal first one, which was indeed not allowed to freely expel its products of reaction. The Raman data were supported by the $m$-line measurements: It was not possible to reproduce the five effective indices measured at $543.5 \mathrm{~nm}$ in the gradedindex waveguide by using the values of thickness and refrac-

TABLE I. Parameters of the three layered sol-gel silica-titania waveguide (SGW1) and of the three reference waveguides (R1, R2, and R3): Chemical composition, thickness $(t)$, refractive index at $543.5(n)$, number of TE and TM modes $(N)$, thickness $\left(t_{i}\right)$, and refractive indices $\left(n_{i}\right)$ of the three layers used for reproducing the $m$-line results.

\begin{tabular}{lccccc}
\hline \hline Composition & $t(\mu \mathrm{m})$ & $n$ & $N$ & $t_{i}$ & $n_{i}$ \\
\hline $\mathrm{R} 1\left(8 \% \mathrm{TiO}_{2}-92 \% \mathrm{SiO}_{2}\right)$ & $1.15 \pm 0.10$ & $1.496 \pm 0.010$ & 1 & 1.0 & 1.478 \\
$\mathrm{R} 2\left(16 \% \mathrm{TiO}_{2}-84 \% \mathrm{SiO}_{2}\right)$ & $0.78 \pm 0.05$ & $1.556 \pm 0.005$ & 2 & 0.78 & 1.556 \\
$\mathrm{R} 3\left(24 \% \mathrm{TiO}_{2}-76 \% \mathrm{SiO}_{2}\right)$ & $0.89 \pm 0.05$ & $1.621 \pm 0.005$ & 2 & 0.89 & 1.621 \\
\hline \hline
\end{tabular}




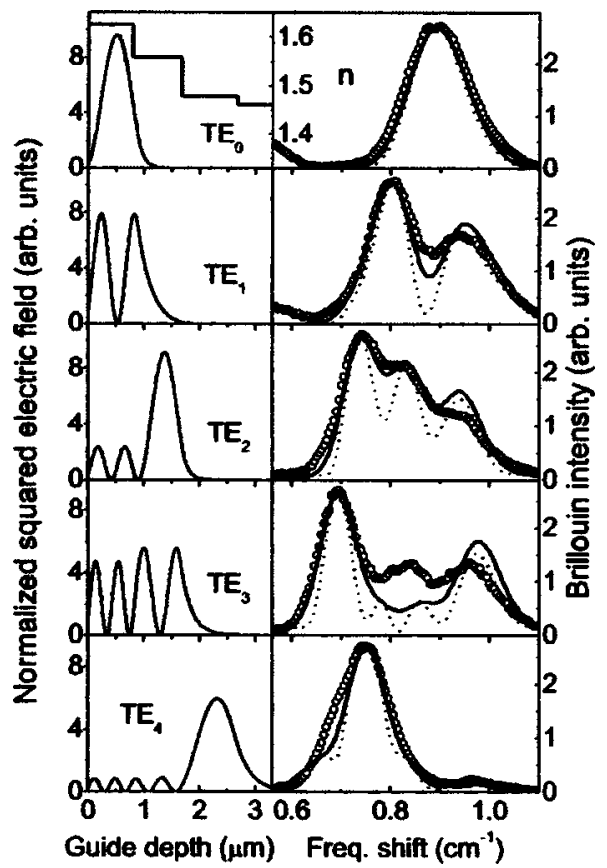

FIG. 6. Upper left-hand side frame: Refractive index profile at $514.5 \mathrm{~nm}$ of the three-layered SGW1 planar waveguide. Left-hand side column: Calculated squared electric-field patterns of the five $\mathrm{TE}_{m}$ modes. Right-hand side column: BS (open circles), calculated spectra (dotted line), and convolution of the calculated spectra with the instrumental response (solid line). The longitudinal sound velocity used in the fit is $v_{L}=5.9 \mathrm{~km} / \mathrm{s}$ for $m=0,1,2$, and 3 and $v_{L}=5.75 \mathrm{~km} / \mathrm{s}$ for $m=4$.

tive index measured in the three reference waveguides. A refractive index lower than that expected for the $8 \% \mathrm{TiO}_{2}-92 \% \mathrm{SiO}_{2}$ composition was needed for reproducing, in particular, the $\mathrm{TE}_{4}$ mode, which has a large field in this layer. Therefore, we were forced to take, as free parameters, the thickness and refractive index of this layer (see Table I), in this way reproducing the $m$-line results within the experimental error. The refractive index profile, calculated at $514.5 \mathrm{~nm}$ by extrapolation of the data at $543.5 \mathrm{~nm}$ and at $632.8 \mathrm{~nm}$, is shown on the upper left-hand side of Fig. 6. The calculated profiles of the squared electric field show that the different modes selectively excite the different layers of the waveguide: The $\mathrm{TE}_{0}$ mode is practically completely confined in the third (from the substrate) layer, while the $\mathrm{TE}_{4}$ mainly occupies the first layer. The BS in the longitudinal phonon spectral region are shown on the right-hand side of Fig. 6, together with the calculated ones, after averaging the Stokes and anti-Stokes spectra and subtracting the transverse phonon contribution. The longitudinal phonon contribution of the $\mathrm{TE}_{2}$ spectrum shows a feature with three peaks of comparable intensities. The phenomenology is very similar to that of the $\mathrm{TE}_{2}$ spectrum of the triangular refractive index profile studied in Ref. 12. In such a system, the calculation made a provision for the presence of $m+1$ peaks of comparable intensity, for any $m$, instead of the two peaks typical of the step profiles. ${ }^{11}$ For the $\mathrm{TE}_{0}, \mathrm{TE}_{1}$, and $\mathrm{TE}_{2}$ spectra, the agreement is good even if the observed intensity of the higher-frequency components in the $\mathrm{TE}_{1}$ and $\mathrm{TE}_{2}$ spectra is lower than expected and the observed linewidth is slightly larger than expected. In the $\mathrm{TE}_{3}$ spectrum, only three peaks

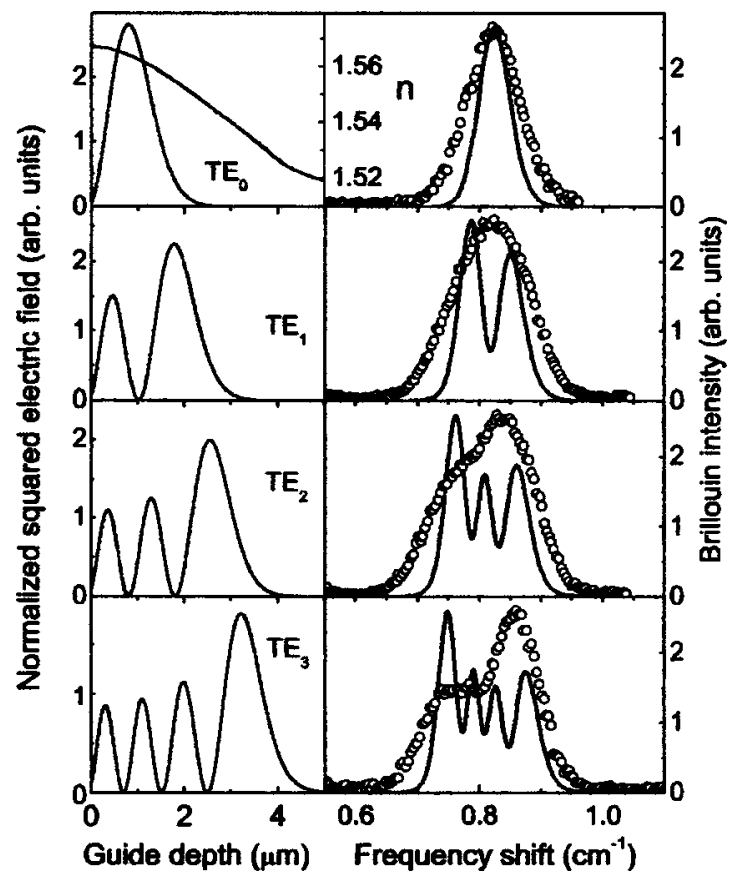

FIG. 7. Upper left-hand side frame: Refractive index profile at $514.5 \mathrm{~nm}$ of the IEW1 waveguide. Left-hand side column: Calculated squared electricfield patterns of the four $\mathrm{TE}_{m}$ modes. Right-hand side column: Experimental (open circles) and calculated (solid line) Brillouin spectra. The longitudinal sound velocity used in the calculation are $v_{L}=5.68 \mathrm{~km} / \mathrm{s}$ for $m=0, v_{L}$ $=5.70 \mathrm{~km} / \mathrm{s}$ for $m=1,2$, and $v_{L}=5.75 \mathrm{~km} / \mathrm{s}$ for $m=3$.

are observed instead of the four calculated ones. The $\mathrm{TE}_{4}$ shows a single strong peak with shoulders, partially reproduced by the calculations. It is possible that the lack of complete agreement of the model results with the experimental $\mathrm{TE}_{3}$ and $\mathrm{TE}_{4}$ spectra is due to bad modeling of the first layer. In any case, a general agreement is present in all spectra, sufficient to determine with high accuracy the longitudinal sound velocity. Within the experimental error, we use the same value $v_{L} \simeq 5.9 \mathrm{~km} / \mathrm{s}$ for the four excitation with $m \leqslant 3$. These four modes involve, with different weights, the two external layers with the compositions $24 \% \mathrm{TiO}_{2}-76 \% \mathrm{SiO}_{2}$ and $16 \% \mathrm{TiO}_{2}-84 \% \mathrm{SiO}_{2}$. We should conclude that the longitudinal sound velocity is nearly the same for the two compositions. A rather weak dependence of the sound velocity on the titania content was already observed. ${ }^{11,26}$ On the contrary, the $\mathrm{TE}_{4}$ spectrum, with most of the excitation in the internal layer with the composition of $8 \% \mathrm{TiO}_{2}-92 \% \mathrm{SiO}_{2}$, shows a lower value of the longitudinal sound velocity, $v_{L}$ $\simeq 5.75 \mathrm{~km} / \mathrm{s}$. Therefore, the Brillouin scattering result is in agreement with those of the measurements of the refractive and of Raman scattering, all indicating that this internal layer has a residual porosity and did not undergo full densification.

The BS of transverse phonons is well resolved from the longitudinal contribution and the tail of the Rayleigh scattering only for the excitation in $\mathrm{TE}_{0}$ and $\mathrm{TE}_{1}$ modes. The two spectra are fitted with $v_{T}=3.6 \mathrm{~km} / \mathrm{s}$.

\section{D. lon-exchanged waveguides}

Figure 7 shows the result for a Ag-exchanged soda-lime waveguide (IEW1). The refractive index profile at $514.5 \mathrm{~nm}$, 
reported in the upper left-hand side frame, was obtained by extrapolation of the profiles at $632.8 \mathrm{~nm}$ and $543.5 \mathrm{~nm}$. The graded-index profile is caused by the exchange of $\mathrm{Na}^{+}$ions with heavier $\mathrm{Ag}^{+}$ions and reflects the $\mathrm{Ag}^{+}$concentration, which extends to about $3 \mu \mathrm{m}$ in the glass. ${ }^{27}$ The observed BS show quite broad peaks, much broader than the calculated ones and broader than those of the experimental resolution. The expected splitting of the $\mathrm{TE}_{1}$ spectrum is not observed and only two broad peaks, not well resolved, appear in the $\mathrm{TE}_{2}$ and $\mathrm{TE}_{3}$ spectra, instead of the three and four expected, respectively. Furthermore, the low-frequency component is weaker than the high-frequency one, whereas the calculation makes the opposite provision. Very similar results were obtained on other Ag-exchanged soda-lime waveguides, including a thicker waveguide with ten TE modes at $514.5 \mathrm{~nm} .{ }^{28}$ The simple model, which does not account for phonon refraction and reflection, does not accurately reproduce the spectra in these waveguides with a gradient in the elastic constants. In any case, the comparison of the calculated spectra with the observed ones provides interesting information. The best agreement, even if there is some degree of arbitrariness in the superposition of the measured and calculated spectra, is obtained with different values of the sound velocity for the different TE modes. The value of the effective sound velocity seems to increase with the $m$-modal index, toward the bulk value $v_{L}=5.9 \mathrm{~km} / \mathrm{s}$. Looking at the excitation profiles reported on the left-hand side of Fig. 7, we conclude that the exchange of sodium with silver lowers the sound velocity of the glass as expected since the mass density of the glass should increase.

\section{CONCLUSIONS}

BS produced by bulk phonons have been detected in waveguides of different thicknesses and compositions, including graded-index waveguides, with the excitation in a mode of the waveguide. The shapes of the BS depend on the mode index and are structured. We can account for the main features of the spectra by means of a simple model which assumes a single-sound velocity for the whole system. The fit to the experimental spectra provides an effective sound velocity relative to the excited layer of the waveguide. In a multimode waveguide, if the modes propagate at different depths, different values of the effective sound velocity are obtained. This allows information to be obtained on the elastic properties of different layers. In a sol-gel derived waveguide, a residual porosity in an internal layer was evidenced by measuring a longitudinal sound velocity lower than that of the densified glass. By comparing the effective longitudinal sound velocities at different depths in an $\mathrm{Ag}^{+}$-exchanged soda-lime waveguide, we showed that the exchange of sodium with silver lowers the value of the sound velocity.

\section{ACKNOWLEDGMENTS}

This research was performed in the context of the following projects: (No. CNR-ICCTI 2001-2004) Optical Am- plification in Rare-Earth-Doped Glass Integrated Optical Waveguides, (No. CNR-CNRS 2002-2003) Improvement of the Multitarget rf Sputtering and Sol-Gel Techniques for Fabrication of $\mathrm{Er}^{3+}-\mathrm{Yb}^{3+}$ Silica-Based Photonic Components Operating in the C-Telecommunication Band, and (No. COFIN 2002) Materiali Nanostrutturati per l'Ottica Integrata.

${ }^{1}$ D. Heiman, D. S. Hamilton, and R. W. Hellwarth, Phys. Rev. B 19, 6583 (1979).

${ }^{2}$ J. R. Sandercock, in Light Scattering in Solids, edited by M. Cardona and G. Guntherodt (Springer, Berlin, 1982).

${ }^{3}$ F. Nizzoli and J. R. Sandercock, in Dynamical Properties of Solids, edited by G. Horton and A. A. Maradudin (North-Holland, Amsterdam, 1990), p. 281.

${ }^{4}$ P. Mutti, C. E. Bottani, G. Ghislotti, M. Beghi, G. A. D. Briggs, and J. R. Sandercock, in Advances in Acoustic Microscopy, edited by G. A. D. Briggs (Plenum, New York, 1995), p. 249.

${ }^{5}$ A. C. Ferrari, J. Robertson, M. G. Beghi, C. E. Bottani, R. Ferulano, and R. Pastorelli, Appl. Phys. Lett. 75, 1893 (1999).

${ }^{6}$ J. R. Sandercock, Phys. Rev. Lett. 29, 1735 (1972).

${ }^{7}$ N. L. Rowell, V. C.-Y. So, and G. I. Stegeman, Appl. Phys. Lett. 32, 154 (1978).

${ }^{8}$ N. L. Rowell and G. I. Stegeman, Phys. Rev. Lett. 41, 970 (1978).

${ }^{9}$ N. L. Rowell, V. C.-Y. So, R. Normandin, and G. I. Stegeman, Can. J. Phys. 60, 1821 (1982).

${ }^{10}$ J. R. Sandercock, Top. Appl. Phys. 51, 173 (1982).

${ }^{11}$ M. Montagna, M. Ferrari, F. Rossi, F. Tonelli, and C. Tosello, Phys. Rev. B 58, R547 (1998).

${ }^{12}$ A. Chiasera, M. Montagna, F. Rossi, and M. Ferrari, J. Appl. Phys. 94, 4876 (2003).

${ }^{13}$ Integrated Optical Circuits and Components. Design and Applications edited by E. J. Murphy (Marcel Dekker, New York, 1999).

${ }^{14}$ Opt. Eng. 37, 1100 (1998), (Speical issue).

${ }^{15}$ J. D. Shephard, D. Furniss, P. A. Houston, and A. B. Seddon, J. Non-Cryst. Solids 284, 160 (2001).

${ }^{16}$ A. Bartolotta, G. Carini, G. D'Angelo, M. Ferrari, A. Fontana, M. Montagna, F. Rossi, and G. Tripodo, J. Non-Cryst. Solids 280, 249 (2001).

${ }^{17}$ F. Terki, J. Pelous, P. Dieudonne, and T. Woignier, J. Non-Cryst. Solids 225, 277 (1998).

${ }^{18}$ C. Tosello, F. Rossi, S. Ronchin, R. Rolli, G. C. Righini, F. Pozzi, S. Pelli, M. Fossi, E. Moser, M. Montagna, M. Ferrari, C. Duverger, A. Chiappini, and C. De Bernardi, J. Non-Cryst. Solids 284, 230 (2001).

${ }^{19}$ S. Ronchin, A. Chiasera, M. Montagna, R. Rolli, C. Tosello, S. Pelli, G. C. Righini, R. R. Gonçalves, S. Ribeiro, C. De Bernardi, F. Pozzi, C. Duverger, R. Belli, and M. Ferrari, Proc. SPIE 4282, 31 (2001).

${ }^{20}$ L. Zampedri, M. Ferrari, C. Armellini, F. Visintainer, C. Tosello, S. Ronchin, R. Rolli, M. Montagna, A. Chiasera, S. Pelli, G. C. Righini, A. Monteil, C. Duverger, and R. R. Gonçalves, J. Sol-Gel Sci. Technol. 26, 1033 (2003).

${ }^{21}$ S. Pelli and G. C. Righini, Advances in Integrated Optics, edited by M. Martellucci, A. N. Chester, and M. Bertolotti (Plenum, New York, 1994), p. 1.

${ }^{22}$ K. S. Chiang, J. Lightwave Technol. 3, 385 (1985).

${ }^{23}$ C. Tosello, F. Rossi, S. Ronchin, R. Rolli, G. C. Righini, F. Pozzi, S. Pelli, M. Fossi, E. Moser, M. Montagna, M. Ferrari, C. Duverger, A. Chiappini, and C. De Bernardi, J. Non-Cryst. Solids 284, 230 (2001).

${ }^{24}$ C. Duverger, M. Montagna, R. Rolli, S. Ronchin, L. Zampedri, M. Fossi, S. Pelli, G. C. Righini, A. Monteil, C. Armellini, and M. Ferrari, J. NonCryst. Solids 280, 261 (2001).

${ }^{25}$ M. Ferrari, M. Montagna, S. Ronchin, F. Rossi, and G. C. Righini, Appl. Phys. Lett. 75, 1529 (1999).

${ }^{26}$ K. Hirao, K. Tanaka, S. Furukawa, and N. Soga, J. Mater. Sci. Lett. 14, 697 (1995).

${ }^{27}$ G. C. Righini, S. Pelli, M. Brenci, M. Ferrari, C. Duverger, M. Montagna, and R. Dall'Igna, J. Non-Cryst. Solids 284, 223 (2001).

${ }^{28}$ M. Montagna, S. Ronchin, F. Rossi, C. Tosello, E. Moser, M. Ferrari, and G. C. Righini, Proc. SPIE 3942, 224 (2000). 